\title{
Toward High Energy Neutrino Detection with the Radar Echo Telescope for Cosmic Rays (RET-CR)
}

\author{
Steven Prohira ${ }^{a * *}$ and Krijn D de Vries $^{b}$ on behalf of the Radar Echo Telescope \\ Collaboration \\ (a complete list of authors can be found at the end of the proceedings) \\ ${ }^{a}$ Department of Physics, Center for Cosmology and AstroParticle Physics (CCAPP), The Ohio State \\ University, Columbus $\mathrm{OH}$, USA \\ ${ }^{b}$ Vrije Universiteit Brussel, Brussel, Belgium \\ E-mail: prohira.1@osu.edu, Krijn.de.Vries@vub.be
}

The Radar Echo Telescope for Cosmic Rays (RET-CR) is a pathfinder experiment for the Radar Echo Telescope for Neutrinos (RET-N), a next-generation in-ice detection experiment for ultra high energy neutrinos. RET-CR will serve as the testbed for the radar echo method to probe high-energy particle cascades in nature, whereby a transmitted radio signal is reflected from the ionization left in its wake. This method, recently validated at SLAC experiment T576, shows promising preliminary sensitivity to neutrino-induced cascades above the energy range of optical detectors like IceCube. RET-CR intends to use an in-nature test beam: the dense, in-ice cascade produced when the air shower of an ultra high energy cosmic ray impacts a high-elevation ice sheet. This in-ice cascade, orders of magnitude more dense than the in-air shower that preceded it, is similar in profile and density to the expected cascade from a neutrino-induced cascade deep in the ice. RET-CR will be triggered using surface scintillator technology and will be used to develop, test, and deploy the hardware, firmware, and software needed for the eventual RET-N. We present the strategy, status, and design sensitivity of RET-CR, and discuss its application to eventual neutrino detection.

$37^{\text {th }}$ International Cosmic Ray Conference (ICRC 2021)

July 12 th - 23rd, 2021

Online - Berlin, Germany

\footnotetext{
${ }^{*}$ Presenter
} 


\section{Introduction}

Detection of the low flux of neutrinos at energies beyond $10^{16} \mathrm{eV}$ requires technologies that can cover large detection volumes with a minimum of apparatus [1-7]. In this article, we will discuss a pathfinder experiment that uses the radar echo method, a radio-based technique that may compliment existing strategies and help to extend the energy range of detected neutrinos up beyond $10 \mathrm{PeV}$.

The radar echo method was first put forward as a candidate detection strategy for ultra high energy (UHE) particles in the early 1940s, when it was first proposed to explain anomalous radio echoes from the upper atmosphere [8]. It was theorized that these echoes could be from radar reflecting from the charged tracks left behind as a cosmic ray produces a cascade in the atmosphere. It turns out that these particular echoes were actually caused by meteors, but the idea took hold, and multiple experimental efforts to date have searched for radar reflections from UHE cosmic rays [9-12].

These efforts have not made a detection, primarily because the in-air ionization densities are too low, the free electron lifetime is too short, and the rate of collisions between free electrons and the neutral atoms in the air is too high. All together, it makes it so that in-air detection via radar echo is not feasible.

Detection in the ice, however, is a different situation. A UHE neutrino interacting in the ice would produce a similar cascade to those in air, but 1000 times shorter and more dense, owing to the increased relative density of ice to air. This increased ionization density makes the cascade detectable, and has renewed interest in the radar echo method. The technique has been recently validated in the lab [13], and in this proceeding we describe the next stepping stone toward a full scale neutrino detector.

When a UHE cosmic ray interacts in the atmosphere it produces a cascade and subsequent cloud of ionization $[14,15]$. This in-air cloud is not detectable via radar, as above. However, if the elevation of the ground is high, a significant fraction of the primary energy will hit the ground. If that ground is a radio-transparent medium like ice, then the dense in-ice cascade from the cosmic ray air shower may be detectable via radar echo. It is this in-ice cascade that we aim for with the Radar Echo Telescope for Cosmic Rays (RET-CR), where the radar echo method will be tested in nature with a natural source. Such high-elevation ice sheets can be found in the Arctic and Antarctic, with some in the latter rising to over $3 \mathrm{~km}$, sufficiently high for $\gtrsim 10 \%$ of the primary energy to reach the surface (for primary energies greater than $10 \mathrm{PeV}$ and zenith angles below roughly 30 degrees).

\section{Experimental Concept}

The experimental concept is shown in Figure 1. The charged particles from the in-air component of the cosmic ray air shower will trigger a surface system, telling the radar data acquisition system to capture an event. The in-ice radar system will run continuously, and will be a testbed for a variety of radar-based triggers for an eventual neutrino detector.

The surface system is comprised of an array of detectors with scintillator panels and radio antennas to detect the in-air portion of the cosmic ray cascade, based on the detectors from the CODALEMA experiment $[16,17]$. Virtually every cascade with a primary energy above $16.5 \mathrm{PeV}$ 


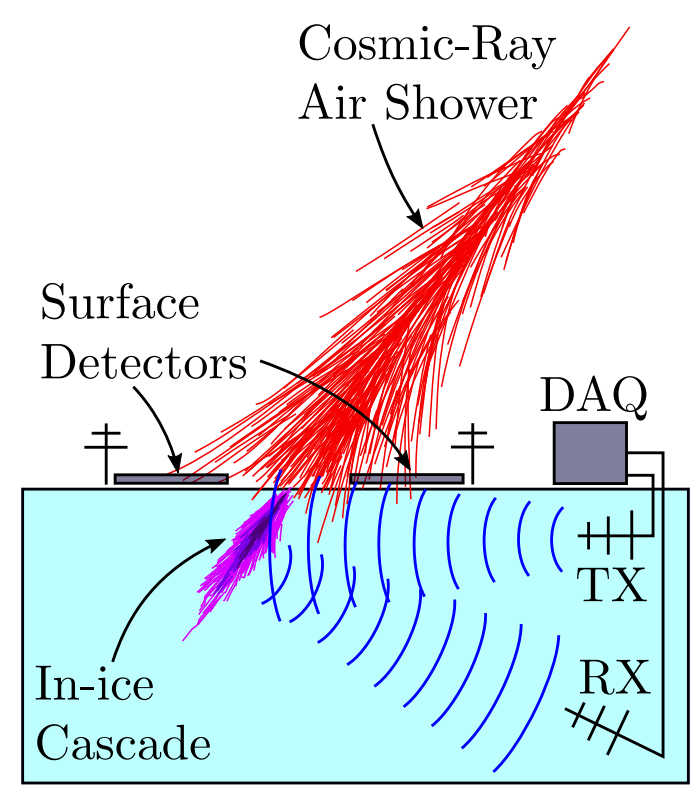

Figure 1: The RET-CR concept. A surface detector triggers on the charged in-air cascade particles as they reach the surface. The energy of this in-air cascade is deposited into the ice, where a denser in-ice cascade is produced. Radio is broadcast from the transmitter (TX) and reflected from the in-ice cascade to the embedded receiver $(\mathrm{RX})$.

will trigger the surface system, initiating a radar readout. Information from this surface system about cascade location and zenith will assist in training radar reconstruction algorithms.

The in-ice radar system will consist of an 8 antenna phased transmitter, and 8 receivers as shown in Figure 8 of Ref. [18]. The receivers and transmitters will have azimuthal symmetry, with a high-gain region just below the surface of the ice, where we expect the cascades to occur after the UHE cosmic ray cascade has crossed the air/ice boundary. At the heart of the transmitter and data acquisition system will be a Xilinx RF Solution on Chip (RFSoC), with integrated FPGA, CPU, 8 channel analog-to-digital conversion and 8 channel digital-to-analog conversion.

The system will run autonomously on solar power, and have communications capabilities for remote commanding and telemetry. Local storage will provide for data capture. The instrument will operate during the austral summer, with Taylor Dome, Antarctica, being our primary location at time of writing.

\section{Simulation}

The experiment has been simulated in detail [18] using a 3-part simulation chain involving Corsika [19], GEANT4[20], and RadioScatter [21, 22]. Primary cosmic rays were simulated at a variety of energies and zenith angles. The in-ice cascade from a subset of these energies and angles (due to computational requirements, not all cascades could be simulated) in order to generate a distribution of the deposited energy For further details of this simulation chain, please see Ref. [18] and our other proceeding from this conference, "Simulation and Optimisation for the Radar Echo Telescope for Cosmic Rays". 


\section{Event Rate}

The expected event rate for cosmic rays with energies above $10^{17} \mathrm{eV}$ is roughly 1 per day, as shown in Figure 2. We hope to detect a number of cosmic rays at a variety of energies, positions, and arrival angles, in order to study the radar echo signal and train our trigger techniques.

The radar signal itself has many interesting properties that may be probed to achieve useful angular and energy resolution. For details on the signal properties of the radar echo signal, please see another proceeding from this conference, "Investigating signal properties of UHE particles using in-ice radar for the RET experiment".

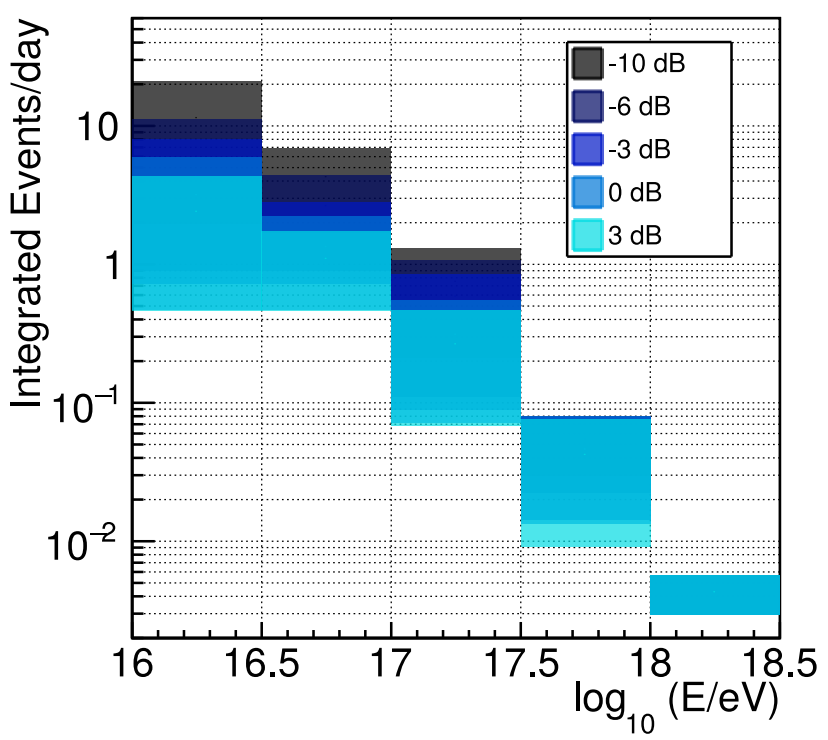

Figure 2: The integrated daily event rate for RET-CR. Each bin represents the total number of events per day at that bin's energy and above, for the indicated SNR.

\section{Conclusion}

The Radar Echo Telescope for Cosmic Rays is a pathfinder experiment to test the radar echo method of high-energy particle detection in nature. A deployment to the polar regions will culminate in the detection of in-ice cascades produced by UHE cosmic ray cascades after they cross the air/ice boundary. The trigger routines and signal properties studied with RET-CR will be used for our eventual neutrino telescope, the Radar Echo Telescope for Neutrinos (RET-N).

\section{Acknowledgements}

RET-CR is supported by the National Science Foundation under award numbers NSF/PHY2012980 and NSF/PHY-2012989. This work is also supported by the Flemish Foundation for Scientific Research FWO-12ZD920N, the European Research Council under the EU-ropean Unions Horizon 2020 research and innovation programme (grant agreement No 805486), and the Belgian 
Funds for Scientific Research (FRS-FNRS). A. Connolly acknowledges support from NSF Award \#1806923. S. Wissel was supported by NSF CAREER Awards \#1752922 and \#2033500. DZB is grateful for support from the U.S. National Science Foundation-EPSCoR (RII Track-2 FEC, award ID 2019597). We express our gratitude to R. Dallier, L. Martin, J-L. Beney and the CODALEMA experiment for providing electronics and hardware to be used in the surface radio stations of RET-CR. Computing resources were provided by the Ohio Supercomputer Center.

\section{References}

[1] P. Allison et al., Design and Initial Performance of the Askaryan Radio Array Prototype EeV Neutrino Detector at the South Pole, Astropart. Phys. 35 (2012) 457 [1105 . 2854].

[2] S.R. Klein et al., A radio detector array for cosmic neutrinos on the ross ice shelf, IEEE Transactions on Nuclear Science 60 (2013) 637.

[3] ANITA collaboration, The Antarctic Impulsive Transient Antenna Ultra-high Energy Neutrino Detector Design, Performance, and Sensitivity for 2006-2007 Balloon Flight, Astropart. Phys. 32 (2009) 10 [0812 . 1920].

[4] S. Wissel et al., Prospects for high-elevation radio detection of $>100 \mathrm{PeV}$ tau neutrinos, JCAP 11 (2020) 065 [2004.12718].

[5] J.A. Aguilar et al., The Next-Generation Radio Neutrino Observatory-Multi-Messenger Neutrino Astrophysics at Extreme Energies, 1907.12526.

[6] Q. Abarr et al., The Payload for Ultrahigh Energy Observations (PUEO): A White Paper, 2010.02892.

[7] A.V. Olinto et al., POEMMA: Probe Of Extreme Multi-Messenger Astrophysics, PoS ICRC2017 (2018) 542 [1708.07599].

[8] A.C.B. Lovell, Reminiscences and discoveries: The blackett-eckersley-lovell correspondence of world war ii and the origin of jodrell bank, Notes and Records of the Royal Society of London 47 (1993) 119.

[9] P. Blackett and C. Lovell, Radio echoes and cosmic ray showers, Proc. Roy. Soc A 177 (1941) 183.

[10] T. Matano, M. Nagano, K. Suga and G. Tanahashi, Tokyo large air shower project, Canadian Journal of Physics 46 (1968) S255.

[11] R. Abbasi et al., Telescope Array Radar (TARA) Observatory for Ultra-High Energy Cosmic Rays, Nucl. Instrum. Meth. A767 (2014) 322 [1405.0057].

[12] R.U. Abbasi et al., First Upper Limits on the Radar Cross Section of Cosmic-Ray Induced Extensive Air Showers, Astropart. Phys. 87 (2017) 1 [1603.05217]. 
[13] S. Prohira et al., Observation of Radar Echoes From High-Energy Particle Cascades, Phys. Rev. Lett. 124 (2020) 091101 [1910. 12830].

[14] P. Auger, Extensive cosmic-ray showers, Review of Modern Physics 11 (1939) 288.

[15] P. Gorham, On the possibility of radar echo detection of ultrahigh-energy cosmic ray induced and neutrino induced extensive air showers, Astropart. Phys. 15 (2001) 177 [hep-ex/0001041].

[16] B. Revenu, D. Charrier, R. Dallier, A. Escudie, D. García-Fernández, A. Lecacheux et al., The CODALEMA/EXTASIS experiment: a multi-scale and multi-wavelength instrument for radio-detection of extensive air-showers, PoS ICRC2017 (2018) 416.

[17] K. Mulrey et al., On the cosmic-ray energy scale of the LOFAR radio telescope, JCAP 11 (2020) 017 [2005 . 13441].

[18] S. Prohira et al., The Radar Echo Telescope for Cosmic Rays: Pathfinder Experiment for a Next-Generation Neutrino Observatory, 2104.00459.

[19] D. Heck, J. Knapp, J. Capdevielle, G. Schatz, T. Thouw et al., Corsika: A monte carlo code to simulate extensive air showers, Report fzka 6019 (1998).

[20] S. Agostineli et al., Geant4-a simulation toolkit, Nuclear Instruments and Methods in Physics Research A 506 (2003) 250.

[21] S. Prohira and D. Besson, Particle-level model for radar based detection of high-energy neutrino cascades, Nucl. Instrum. Meth. A922 (2019) 161 [1710.02883].

[22] S. Prohirahttps://github.com/prchyr/RadioScatter (2017). 


\section{Full Authors List: Radar Echo Telescope Collaboration}

S. Prohira ${ }^{1}$, K.D. de Vries ${ }^{2}$, P. Allison ${ }^{1}$, J. Beatty ${ }^{1}$, D. Besson ${ }^{3,4}$, A. Connolly ${ }^{1}$, P. Dasgupta ${ }^{5}$, C. Deaconu ${ }^{6}$, S. De Kockere ${ }^{2}$,

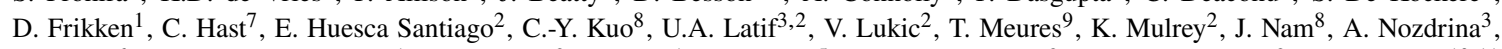
E. Oberla ${ }^{6}$, J.P. Ralston C. Sbrocco ${ }^{1}$, R.S. Stanley ${ }^{2}$, J. Torres ${ }^{1}$, S. Toscano ${ }^{5}$, D. Van den Broeck ${ }^{2}$, N. van Eijndhoven ${ }^{2}$, and S. Wissel ${ }^{10,11}$

${ }^{1}$ Department of Physics, Center for Cosmology and AstroParticle Physics (CCAPP), The Ohio State University, Columbus OH, USA

${ }^{2}$ Vrije Universiteit Brussel, Brussel, Belgium

${ }^{3}$ University of Kansas, Lawrence, KS, USA

${ }^{4}$ National Research Nuclear University, Moscow Engineering Physics Institute, Moscow, Russia

${ }^{5}$ Université Libre de Bruxelles, Brussels, Belgium

${ }^{6}$ Enrico Fermi Institute, Kavli Institute for Cosmological Physics,Department of Physics, University of Chicago, Chicago, IL, USA

${ }^{7}$ SLAC National Accelerator Laboratory, Menlo Park, CA, USA

${ }^{8}$ National Taiwan University, Taipei, Taiwan

${ }^{9}$ University of Wisconsin-Madison, Madison, WI, USA

${ }^{10}$ Departments of Physics and Astronomy \& Astrophysics, Institute for Gravitation and the Cosmos, Pennsylvania State University, University Park, PA, USA

${ }^{11}$ California Polytechnic State University, San Luis Obispo CA, USA 